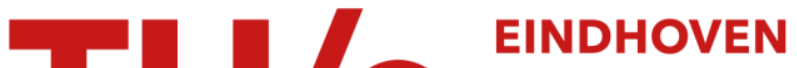 UNIVERSITY OF TECHNOLOGY
}

\section{A pneumatic tactile ring for instantaneous sensory feedback in laparoscopic tumor localization}

\section{Citation for published version (APA):}

Fukuda, T., Tanaka, Y., Kappers, A. M. L., Fujiwara, M., \& Sano, A. (2018). A pneumatic tactile ring for instantaneous sensory feedback in laparoscopic tumor localization. IEEE Transactions on Haptics, 11(4), 485497. [8409323]. https://doi.org/10.1109/TOH.2018.2854753

DOI:

10.1109/TOH.2018.2854753

Document status and date:

Published: 01/10/2018

\section{Document Version:}

Accepted manuscript including changes made at the peer-review stage

\section{Please check the document version of this publication:}

- A submitted manuscript is the version of the article upon submission and before peer-review. There can be important differences between the submitted version and the official published version of record. People interested in the research are advised to contact the author for the final version of the publication, or visit the $\mathrm{DOI}$ to the publisher's website.

- The final author version and the galley proof are versions of the publication after peer review.

- The final published version features the final layout of the paper including the volume, issue and page numbers.

Link to publication

\section{General rights}

Copyright and moral rights for the publications made accessible in the public portal are retained by the authors and/or other copyright owners and it is a condition of accessing publications that users recognise and abide by the legal requirements associated with these rights.

- Users may download and print one copy of any publication from the public portal for the purpose of private study or research.

- You may not further distribute the material or use it for any profit-making activity or commercial gain

- You may freely distribute the URL identifying the publication in the public portal.

If the publication is distributed under the terms of Article 25fa of the Dutch Copyright Act, indicated by the "Taverne" license above, please follow below link for the End User Agreement:

www.tue.nl/taverne

Take down policy

If you believe that this document breaches copyright please contact us at:

openaccess@tue.nl

providing details and we will investigate your claim. 


\title{
A Pneumatic Tactile Ring for Instantaneous Sensory Feedback in Laparoscopic Tumor Localization
}

\author{
Tomohiro Fukuda, Student Member, IEEE, Yoshihiro Tanaka, Member, IEEE, Astrid M.L. Kappers, \\ Michitaka Fujiwara, and Akihito Sano
}

\begin{abstract}
We aim to achieve intraoperative localization of an early-stage gastric tumor that cannot be visually detected during laparoscopic surgery. In this study, we developed and evaluated a pneumatic tactile ring, which is a clinically applicable tactile device to provide instantaneous feedback from a tactile sensor directly manipulated by a surgeon. It was designed to be worn on the finger of the manipulating hand and to present pressure to the finger pad. It is lightweight, cost-effective, disposable, and sterilizable. We also developed a compact pneumatic drive unit to control the pressure and investigated its fundamental performance. The bandwidth of the pressure control was at least $1.3 \mathrm{~Hz}$ with a controllable range of up to $79.7 \mathrm{kPa}$. Moreover, a psychophysical experiment was performed to obtain the Weber ratio of the pressure and evaluate the effectiveness of the instantaneous tactile feedback of the sensor output through the tactile ring. The Weber ratio was 0.40 at the reference pressure of $22.7 \mathrm{kPa}$. The provided tactile feedback significantly reduced the absolute localization error and increased participants' confidence in their answers. It was shown that the tactile feedback through the ring is effective in laparoscopic tumor localization.
\end{abstract}

Index Terms-Tactile display, pneumatic, sensory feedback, tumor localization, laparoscopic surgery.

\section{INTRODUCTION}

$I^{\mathrm{r}}$ NTRAOPERATIVE tumor localization is an important technique in minimally invasive surgery such as laparoscopy. In the laparoscopic resection of early-stage gastric cancer (and intraluminal growing submucosal tumors), surgeons preoperatively obtain information about the tumor from inside the stomach wall under peroral endoscopy. However, preoperative information is not available during surgery, and the early-stage gastric cancer is located on the inner surface of the stomach wall and cannot be visually detected from the outside of the stomach. The surgeon's tactile sense can be used to locate the tumor, which has a different stiffness from the surrounding normal tissue [1], [2], [3]. However, input from the tactile sense is substantially limited in minimally invasive surgery. If the tactile sense were compensated for, the surgeon could perform intraoperative tumor localization by probing the surface of the target tissue.

Many tactile sensors have been developed to achieve intraoperative tumor localization in minimally invasive surgery [4]. Among them, it is popular to measure rich tactile information such as spatially distributed information

- T. Fukuda, Y. Tanaka, and A. Sano are with the Department of Electrical and Mechanical Engineering, Graduate School of Engineering, Nagoya Institute of Technology, Nagoya, Japan.

E-mail: t.fukuda.184@nitech.jp, \{tanaka.yoshihiro, sano\}@nitech.ac.jp.

- T. Fukuda is also Research Fellow of Japan Society for the Promotion of Science, Tokyo, Japan.

- A.M.L. Kappers is with the Department of Human Movement Sciences, Vrije Universiteit Amsterdam, Amsterdam, The Netherlands. E-mail:a.m.l.kappers@vu.nl.

- M. Fujiwara is with the Department of Gastroenterological Surgery, Graduate School of Medicine, Nagoya University, Nagoya, Japan.

E-mail:mfuji@med.nagoya-u.ac.jp.

Manuscript received ${ }^{* *}$; revised ${ }^{* *}$.
[5] and multimodal information (i.e., both applied force and indentation depth) [6]. In addition to the method used to acquire the tactile information, how the information is fed back to the surgeon is an important design factor [7]. One of the means of feedback is via the visual channel; for instance, graphically displaying the sensor output on an additional monitor next to the laparoscopic monitor and overlaying the distributed sensor output on the laparoscopic image. However, these approaches are problematic in clinical applications because the additional monitor will occupy space in the surgical room, and the overlay of the sensor output will degrade the laparoscopic image. It is also possible that overload of the visual channel might be caused [8] because the surgeons always concentrate on the laparoscopic image. Moreover, in palpation with master-slave teleoperation systems, it is common to provide a force to users from a master console according to the measured force [9], [10] or estimated force [11], [12] at the slave side. A major problem when providing such kinesthetic feedback in teleoperation is that the stability of the control loop can be significantly affected [13].

Tactile feedback of the acquired information is a reasonable alternative in surgical situations because it is independent of the visual channel and does not interfere with the surgeon's manipulation. There are many tactile displays that provide sensory feedback to the surgeon in minimally invasive surgery, robot-assisted minimally invasive surgery, and surgical training. Various types of actuation principles have been employed; for instance, pneumatic-driven tactile displays with multiple balloons [14], [15], integrated capacitive sensors for three-axis deformation control [16], and granular jamming chambers to change its stiffness [17] have been developed. Those that supply an air jet to the finger 
pad have also been created [18]. There are tactile displays that use multiple servomotors to actuate a pin array directly [19], [20], [21], to drive a pin array through a flexible tendon and conduit [22], and to control a planar platform [23]. In addition, there are several other principles employed in tactile displays such as the use of shape memory alloys [24], [25], magnetorheological fluid [26], [27], [28], and microhydraulic actuators [29]. These tactile displays aim to supply rich tactile information such as spatially distributed pressure, stiffness, and multiple degrees of freedom of deformation. Although such tactile displays might be intuitive to use, they tend to have complexity due to the need of multiple displaying elements. This might result in high fabrication costs, which impedes their being used as disposable devices as well as their widespread application.

We have focused on temporal information-based palpation, in which a single output is acquired from a sensor and instantaneously fed back to the surgeon. A temporal information-based palpation system has the important advantage of simplicity since only a single sensing element and displaying element are needed unlike systems that use spatially distributed information. Yao et al. have developed a hand-held instrument with an embedded accelerometer and a vibrator to detect small cracks on objects in arthroscopic surgery [30]. This device detects a vibration at its tip while probing objects and presents amplified vibration to the tool body. An assistance device for needle insertion into soft tissues has been developed by Lorenzo et al. [31]. The device detects a force applied at the needle tip and transmits the magnified force to the operator. These studies showed that the detection and display of even a single output was effective in specific tasks. However, these devices were not designed for laparoscopic tumor localization, in which a long and thin probe is manipulated to detect a hard mass within soft tissue. Devices for this purpose should be lightweight and the means of feedback should be appropriately designed so as not to impede the manipulation of the probe. Moreover, some issues regarding their clinical application, such as reduced cost-effectiveness, disposability, and sterilizability, remain.

In a previous study, we developed a forceps-type tactile sensor for laparoscopic tumor localization [32]. The sensor employs an acoustic sensing principle; thus, it has great advantages for surgical applications such as being electrically safe for body tissue, sterilizability, and disposability. The sensor detects a single force applied to the side of the sensor tip. When the surface of the target tissue is continuously scanned by the sensor, the time series of the sensor output includes information about the tumor position. In addition, we have developed a tactile display for the user's foot, where sterilization is not needed, to present a single force related to the sensor output and investigated the effects of visual and tactile feedback from the tactile sensor on ability of the user to detect a phantom tumor and on the user's behavior [33]. It was found that the tactile feedback from the sensor had a positive effect on the sensor manipulation; however, the effect was not significant with respect to tumor detection. This might be because the selected feedback site was the foot, which is quite a distance from the hand manipulating the tactile sensor. The feedback to the foot may, therefore, not be informative enough for

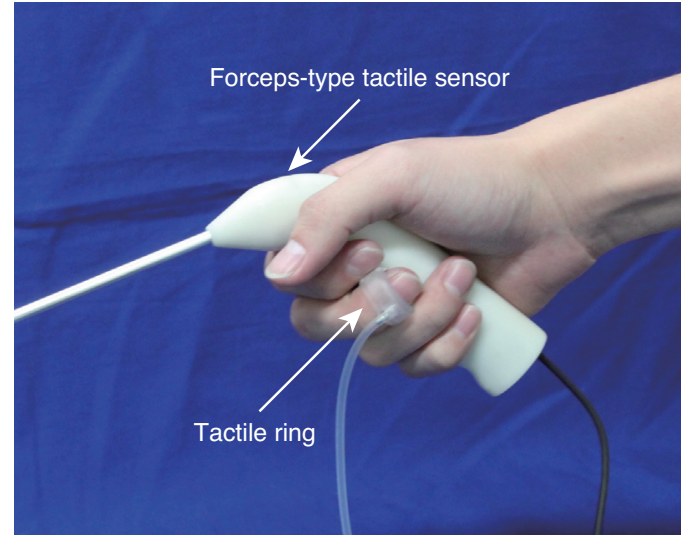

Fig. 1. Tactile ring to provide instantaneous feedback from the forcepstype tactile sensor.

laparoscopic tumor detection. The devices that use only simple information were designed such that a sensor and an actuator are embedded in the same hand-held tool [30], and a needle and manipulation point are coaxially aligned [31]. Spatial coincidence between the manipulating hand and the feedback site might, therefore, facilitate the sensory-motor integration and the interpretation of the given feedback.

The contribution of this study is the development and evaluation of a pneumatic tactile ring, which is a clinically applicable tactile device to provide instantaneous sensory feedback during laparoscopic tumor localization. To address the issue of spatial coincidence, tactile stimulation is transmitted to one of the fingers on the manipulating hand. The tactile ring was designed to present pressure to the surgeon's finger pad (see Fig. 1). Since it is driven by pneumatic power, it is lightweight, cost-effective, disposable, and sterilizable. It also hardly impedes the manipulation of the tactile sensor due to its wearability and means of stimulation. We also developed a compact pneumatic drive unit that consists of a diaphragm pump and a voice coil motor to control the inner pressure of the tactile ring continuously and quickly. The static and dynamic responses for the pressure controlled by the drive unit were investigated. Moreover, we performed a psychophysical experiment with 12 participants who had no medical background. Firstly, just noticeable differences (JNDs) for the pressure were measured to quantitatively evaluate how the tactile ring presents the pressure perceived by the user and validates the signal processing applied to the sensor output. A localization test of a phantom early-stage gastric tumor was then conducted in a simulated laparoscopic setup. Localization performances were compared between the conditions with and without tactile feedback.

The design concept of the tactile ring and the details of the tactile ring and pneumatic drive unit are presented in Section 2. In Section 3, the fundamental characteristics of the devices are presented. The details of the psychophysical experiments are provided in Section 4 and the results of the experiments are given in Section 5. Section 6 provides a discussion on the effectiveness and limitations of the tactile ring. Finally, Section 7 concludes this paper. 


\section{TACTILE RING USING PNEUMATIC POWER}

\subsection{Design concept}

We aimed to develop a tactile device that is worn on the finger of the hand used to manipulate a tactile sensor. Placing the tactile device on the finger results in high spatial coincidence between the manipulating hand and the feedback site. Additionally, the device should not impede the sensor manipulation; in particular, it should not have a weight large enough to affect the sensor manipulation due to its large inertia. Thus, high wearability is required. It is also inevitable to meet requirements for clinical application [7]. Sterilization adaptability is a major factor for a device in contact with the surgeon's hand. Furthermore, a lowcost device means that it can easily be used in a disposable manner. To put an actuator, such as a motor and a vibrator, on an adjacent part of the finger will result in reduced wearability, sterilizability, and disposability. Thus, using pneumatic power is a reasonable choice for the development of a clinically applicable tactile device. Pneumatic power can be generated at a distance and has a high power-tovolume ratio. The body of the tactile device can therefore be lightweight, which causes less interference with the sensor manipulation. In addition, its low-cost fabrication allows for its use as a disposable device.

An important design factor is how a reaction force against the stimulation of the finger pad is grounded. Fingernail-grounded tactile devices are suitable to achieve better wearability than grounded and body-grounded devices [34]. There are many fingernail-grounded devices that achieve high wearability such as those in [35], [36], and [37]. In this study, we designed a pneumatic tactile ring, which is a ring-type device for pressure presentation. A ring is a reasonable shape to use to ground the reaction force. There are relevant studies on the development of tactile devices employing a ring shape to achieve high wearability [38], [39], [40], [41]. However, these devices are not currently applicable to surgical situations because of the disadvantages related to the use of electrical elements. In this study, we designed the tactile ring to be rigid; thus, the surgeon can easily wear the device by just inserting it onto the finger, and the reaction force against the pressure presentation is grounded on the back of the finger pad and the nail. Moreover, we developed a pneumatic drive unit to supply air pressure. Many related studies on pneumatic tactile displays have mainly focused on their body; however, fewer studies have been conducted to reduce the overall size of the driving unit. It is popular to use a commercial air compressor, a tank, and a pneumatic valve, which tend to be bulky and are not optimized for specific applications. We aimed to control the inner pressure of the tactile ring continuously and quickly (at least a bandwidth of $1 \mathrm{~Hz}$ ) with a compact setup.

\subsection{Tactile ring}

The tactile ring is shown on the left side of Fig. 2(a), and its composition is shown in Fig. 2(b). It contains a cavity, which is opened at the inner lower side of the ring. A silicone rubber membrane with a thickness of $0.5 \mathrm{~mm}$ was stuck to the opened portion to form the presenting area of pressure. A sponge was attached to the inner upper side of the ring to address individual differences of finger size. The cavity was connected to a silicone rubber tube with a length of $3 \mathrm{~m}$, and an inner and an outer diameter of $2 \mathrm{~mm}$ and $3 \mathrm{~mm}$, respectively. Another tube end was connected to a luer-lock needle, which can easily be attached to/detached from a luer-lock coupling in the pneumatic drive unit. The total weight and cost of the tactile ring including the rubber tube is approximately $17 \mathrm{~g}$ and US\$ 4.5 , respectively.

\subsection{Pneumatic drive unit}

The pneumatic drive unit is shown on the right side of Fig. 2(a), and its composition is shown in Fig. 2(c). The drive unit mainly consists of a diaphragm pump (DSA-2F-12, DENSO SANGYO Co., Ltd.) and a voice coil motor (AVM2010, Akribis Systems Japan Co., Ltd.). The pump can supply a maximum of approximately $90 \mathrm{kPa}$. The voice coil motor has a force sensitivity of $2.0 \mathrm{~N} / \mathrm{A}$. A silicone rubber sheet with a thickness of $1 \mathrm{~mm}$ was attached to the top of the movable part of the motor. Air is supplied at a constant flow rate to the tactile ring from the pump via an aperture with a diameter of $5 \mathrm{~mm}$. An O-ring with the inner and outer diameters of 4.8 and $8.6 \mathrm{~mm}$, respectively, was attached coaxially to the aperture. The voice coil motor presses the silicone rubber sheet against the aperture with the O-ring. This portion acts as a proportional flow valve because the air leakage rate from the aperture varies according to the pressing force of the motor. The dimensions of the unit are $120 \times 80 \times 43 \mathrm{~mm}$ including the protruding portions.

\section{FundamENTAL CHARACTERISTICS}

\subsection{Procedure}

To investigate the pressure response at the presenting area in the tactile ring, a pressure sensor (AP-13A, KEYENCE Corp.) was connected to the pneumatic drive unit via the silicone rubber tube instead of the tactile ring. The pressure was recorded at a sampling frequency of $1 \mathrm{kHz}$.

The relationship between the input current to the voice coil motor and the inner pressure was investigated. The inner pressure was measured for eight input currents $(0.22,0.45,0.69,0.92,1.15,1.39,1.63$, and $1.86 \mathrm{~A})$. Moreover, the step and frequency responses were measured to assess the dynamic performance of the drive unit. The inner pressure was measured for different step inputs that were switched on at $0.5 \mathrm{~s}$ and off at $2.5 \mathrm{~s}$ with eight target currents. For the measurement of the frequency responses, sinusoidal currents $i_{\text {in }}$ with different frequencies $(0.1-1.0 \mathrm{~Hz}$ with a $0.1 \mathrm{~Hz}$ increment and $1-10 \mathrm{~Hz}$ with a $1 \mathrm{~Hz}$ increment) were used as inputs to the voice coil motor, as follows:

$$
i_{\text {in }}=0.5 I_{\mathrm{A}}\{\sin (2 \pi f t)+1\},
$$

where $f$ is the frequency of the input, $t$ is time, and $I_{\mathrm{A}}$ is the amplitude that corresponds to the target current. The frequency responses were measured for the eight target currents. Then, a gain was calculated according to the following equation:

$$
\text { Gain }=20 \log _{10} \frac{P_{\mathrm{A}}}{P_{\text {static }}},
$$

where $P_{\mathrm{A}}$ is the amplitude of the measured inner pressure, and $P_{\text {static }}$ is the corresponding inner pressure for the static 


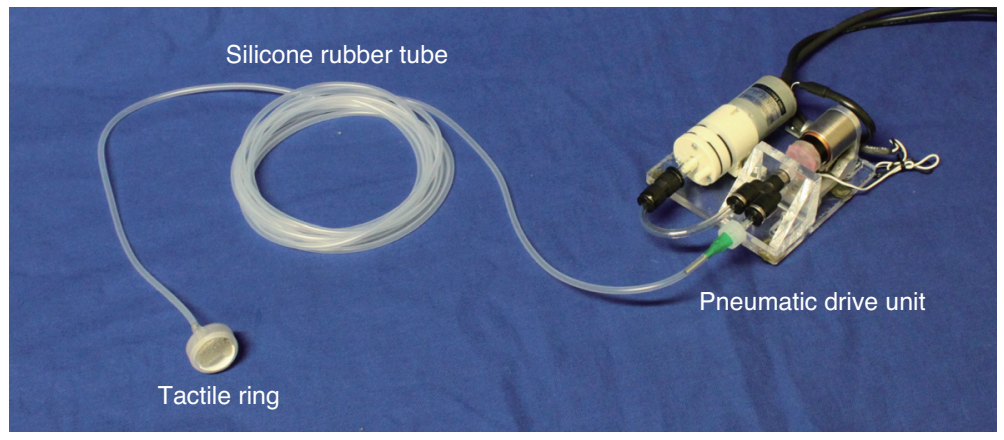

(a)

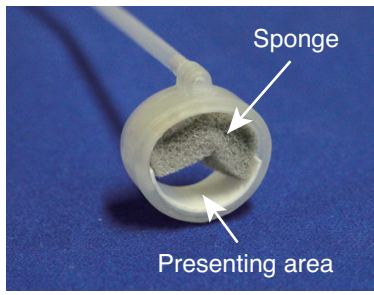

(b)

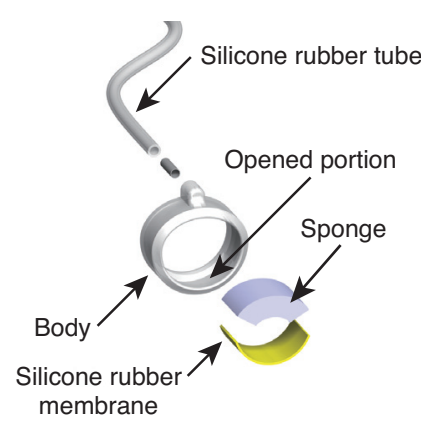

(c)

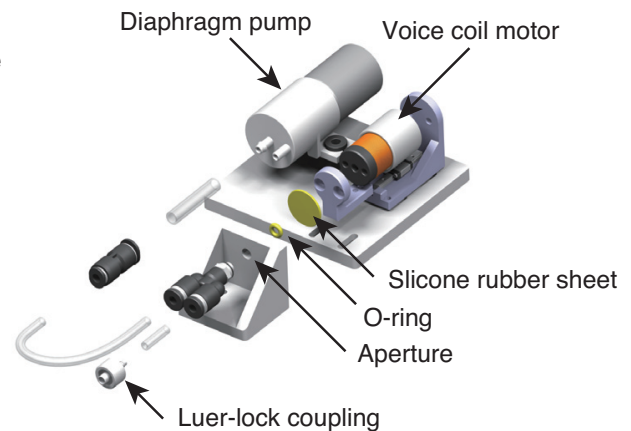

(d)

Fig. 2. Details of the tactile ring using pneumatic power. (a) Overview of the entire system. (b) Close-up of the tactile ring. (c) Expanded view of the tactile ring. (d) Expanded view of the pneumatic drive unit. Note that the scales in the two expanded views are not same.

target current. The gain indicates how the inner pressure decreases in comparison to the static response. All measurements were conducted 10 times for each input current.

\subsection{Results}

Fig. 3(a) shows the relationship between the input current to the voice coil motor and the inner pressure. The error bars indicate the standard deviation of the 10 measurements. The coefficient of determination was 0.999. Fig. 3(b) shows the step responses for different target currents. The mean response curve of 10 measurements is shown for each target. The rising times are longer than the falling times. The rising time, in particular, largely changes according to the target values. The frequency characteristics of the gain for different target currents can be seen in Fig.3(c). The gain decreased by increasing the frequency and also by increasing the target current for frequencies higher than approximately $1 \mathrm{~Hz}$. A bandwidth of the tactile ring was calculated as a limit frequency at which the gain falls below $-3 \mathrm{~dB}$ for each target pressure, and the result is shown in Fig. 3(d). The bandwidth decreased by increasing the target pressure, and the minimum bandwidth was $1.3 \mathrm{~Hz}$ at a target pressure of $79.7 \mathrm{kPa}$.

\subsection{Discussion}

The static relationship between the input current and the inner pressure was almost linear. It is known that the input current to the voice coil motor is linearly related to the force generated. Thus, the inner pressure is related linearly to the pressing force against the aperture. We have assumed that the inner pressure can be calculated from the pressing force and the cross-sectional area of the contact portion between the O-ring and the silicone rubber sheet; that is, the pressing force $F$ is balanced with the force generated by the inner pressure $P$ within the cross-sectional area $A$. In this case, the diameter of the circular cross-sectional area $d$ is calculated as follows:

$$
d=\sqrt{\frac{4 A}{\pi}}=\sqrt{\frac{4 F}{\pi P}}=\sqrt{\frac{4 C_{\mathrm{if}}}{\pi P / I}},
$$

where $C_{\mathrm{if}}=2.0 \mathrm{~N} / \mathrm{A}$ is the force sensitivity of the voice coil motor, and $I$ is the input current to the motor. We calculated an equivalent diameter by substituting the slope of the fitted line (41.3 kPa/A) in Fig.3(a) for $P / I$, and the equivalent diameter was $7.9 \mathrm{~mm}$. The inner and outer diameters of the O-ring were 4.8 and $8.6 \mathrm{~mm}$, respectively. The equivalent diameter lies within them. This indicates that the controllable range of the inner pressure depends on the diameter of the O-ring. Moreover, the maximum suppliable pressure depends on the performance of the diaphragm pump. In this study, a pump that can supply a maximum of approximately $90 \mathrm{kPa}$ was used. It was selected based on related studies on a pneumatic tactile display that presents several tens of $\mathrm{kPa}$ of air pressure [14], [15]. In this way, the static performance of the drive unit can be designed by selecting a diaphragm pump, a voice coil motor, and an $\mathrm{O}$ ring according to its application.

The step responses of the inner pressure depended on the control direction and the target pressure as shown in Fig. 3(b). In particular, the rising response curves for all target pressures overlapped, and the rising time changed according to the target pressure. This is due to the pressure- 


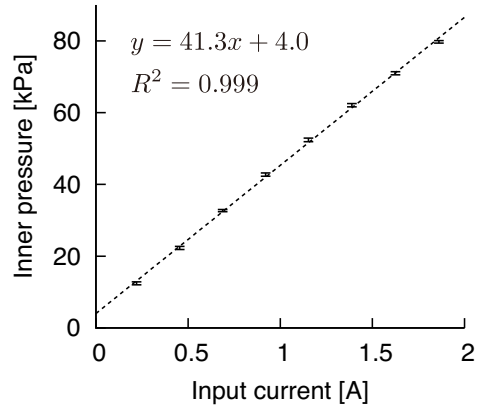

(a)

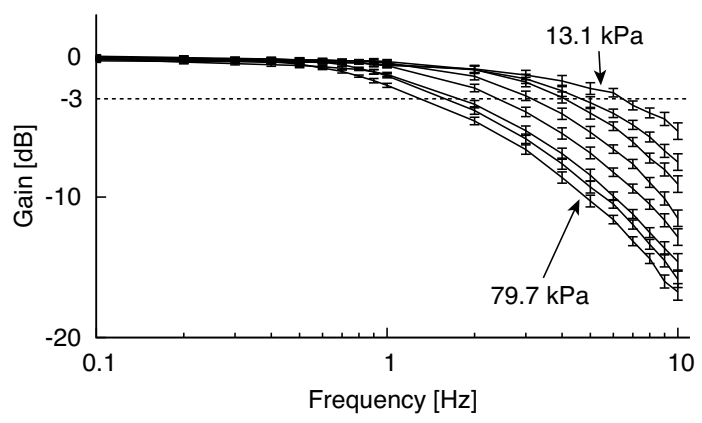

(c)

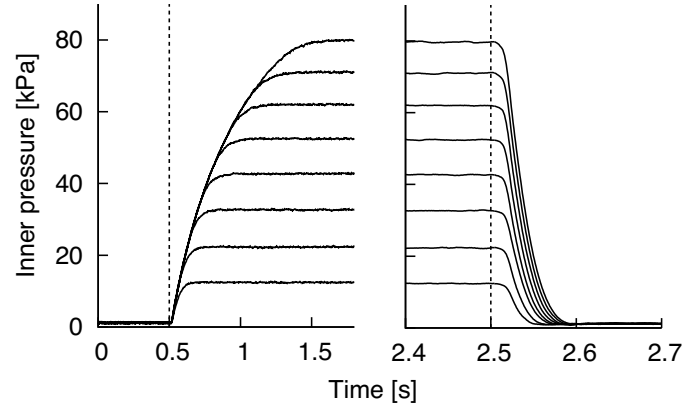

(b)

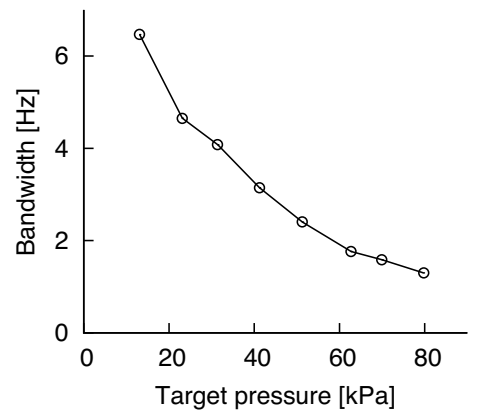

(d)

Fig. 3. Fundamental performances of the tactile ring. (a) Static relationship between the input current and inner pressure. (b) Step responses of the inner pressure for different target currents. Rising and falling responses are shown at different time scales. (c) Frequency responses of the gain of the inner pressure for different target pressures. (d) Bandwidth of the gain for different target pressures.

supply mechanism. The diaphragm pump was directly connected to the tactile ring, and a constant airflow was supplied. Thus, the airflow rate from the diaphragm pump is dominant in the rising responses. On the other hand, the falling responses did not depend as much on the target pressure. This is because the performance of the pump did not affect the falling response, where the air was only discharged. The falling response can, however, be affected by the diameter of the aperture that corresponds to a pneumatic resistance. Thus, the dynamic performance of the drive unit can be designed through the selection of the airflow rate of a diaphragm pump and the diameter of the aperture. Moreover, the inner diameter and the length of the silicone rubber tube strongly affect the dynamic performance [42], and this should be taken into account in the design of the drive unit.

The bandwidth of the device was at least $1.3 \mathrm{~Hz}$. In this study, we aimed to achieve a bandwidth of approximately $1 \mathrm{~Hz}$ since the output of our tactile sensor had frequency components lower than roughly $1 \mathrm{~Hz}$ when the user scanned the surface of a phantom of the stomach wall containing a typical tumor. Although the typically required bandwidth for a pin-array tactile display with $2 \mathrm{~mm}$ spacing is $30 \mathrm{~Hz}$ [43], we did not need a bandwidth this large because our device presents only a single pressure.

\section{PSYCHOPHYSICAL EXPERIMENT}

A psychophysical experiment measuring the JNDs of the presented pressure and a localization task of a phantom tumor in a simulated laparoscopic setup was performed. The measurement of the JNDs aimed to quantitatively evaluate how the tactile ring presents the pressure that can be distinguished by the user and validate the empirically determined signal processing to control the tactile ring. Moreover, we aimed to evaluate how the instantaneous tactile feedback through the tactile ring contributes to tumor localization. In laparoscopic resection of an early-stage gastric tumor, surgeons preoperatively acquire information about the tumor such as its dimensions and position using peroral endoscopy. The surgeons then find the accurate position of the tumor through tactile feedback to determine the appropriate cut-line. Thus, we designed our localization task such that a phantom tumor with constant dimensions was always present, and only its position was changed. This information was provided to the participants before localization trials.

\subsection{Participants}

In this study, we aimed to assess the effectiveness of the tactile ring in a fair manner. Professional surgeons will have a large variation in their surgical skills and experience. Thus, in order to reduce the effect of variation in manipulation skills on our experiment, we employed novice participants and provided them with the same practice trials before the experiment. Twelve persons ( 9 male and 3 female, age range 18-25, mean 20.4) without any medical background were paid to participate in this experiment. Eleven participants out of the twelve were strongly right-handed, and one participant was strongly left-handed according to Coren's test [44]. They gave their written informed consent before participation. The experimental protocol was conducted in accordance with the ethical standards of the Helsinki Declaration and approved by the Ethical Committee of the Nagoya Institute of Technology. 


\subsection{Feedback signal to tactile ring}

We aim to give instantaneous sensory feedback through the tactile ring to the operator of a tactile sensor. We used our proposed tactile sensor employing acoustic sensing principles [32] for the instantaneous feedback (see Fig. 4(a)). The sensor has high surgical applicability since it is electrically safe for body tissue, disposable, and sterilizable. The sensor was somewhat improved from [32] for better usability; for instance, the entire length was $50 \mathrm{~mm}$ longer than the previous device, and the handle of the sensor was redesigned for better grip. In particular, the position of the sensing area was shifted toward the tip of the sensor, which assists the user in scanning the area of interest accurately. The sensor responds to a normal force applied to the sensing area.

The signal processing of the sensor output that controls the display device is an important design factor. We empirically designed the signal processing according to the results of pilot experiments and comments from clinicians who tested our device. The typical sensor output and the feedback signal to the tactile ring are shown in Fig.4(b). The sensor output responds with two small peaks since the target tumor has a toroidal shape. The sensor output was firstly filtered by a band-pass filter with a bandwidth of $0.5-10 \mathrm{~Hz}$ because the tactile ring aimed to present the relative pressure difference during scanning. Then, a gain of $80.7 \mathrm{kPa} / \mathrm{V}$ was applied to the sensor output. However, the gain was reduced to zero over $0.3 \mathrm{~s}$ after the initial contact between the sensor and the target object, and subsequently, it was gradually increased to its original value (see Fig. 4(b)). The initial contact was detected based on a sensor output threshold $(0.01 \mathrm{~V})$. This aspect of the signal processing was added to remove the large pressure feedback due to the initial contact. An offset value corresponding to a pressure of $15.8 \mathrm{kPa}$ was always added, which allowed for a negative feedback signal to be presented. The offset was empirically determined not to be large because, as shown in Fig. 3(d), a large pressure results in a smaller dynamic response of the

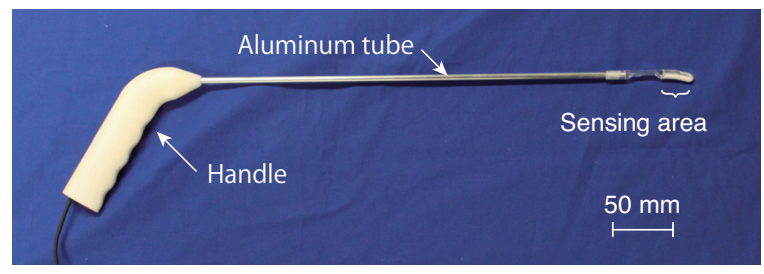

(a)

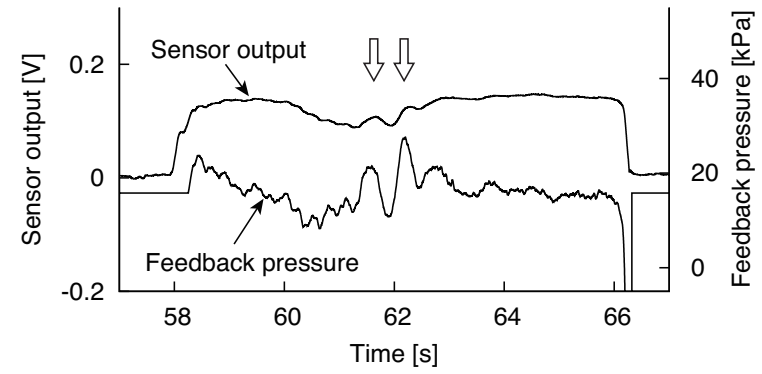

(b)

Fig. 4. (a) Forceps-type tactile sensor used for the localization test. (b) Typical output from the tactile sensor and the feedback pressure. Open arrows indicate two peaks that mark the tumor. pneumatic drive unit. The values of the gain and offset were fixed for all of the participants.

\subsection{Phantom of stomach wall}

We aim to localize a 0-IIc (superficial ulcerative) type of early-stage gastric cancer [45], which is located on the mucosal side of the stomach wall and typically has a toroidal shape. We have fabricated a phantom of the stomach wall with the tumor on the basis of the actual dimensions and stiffness of the stomach and the tumor in our previous study [33]. In this study, although the fabricated phantom was similar, the width was increased to $160 \mathrm{~mm}$ to enable the production of stimuli with different tumor positions (see Fig. 5(a)). A stimulus for the localization task was prepared by putting the phantom with the mucosal side down on a sponge sheet with a thickness of $10 \mathrm{~mm}$. The two ends of the phantom were supported by rigid semicircular bases and fixed by semicylindrical plates (see Fig.5(b)). Stimuli with different tumor positions were prepared by shifting the position of the phantom on the sponge along the $x$-direction.

Two different stimulus sets were prepared for practices and localization trials to prevent participants from memorizing the number or the position of the stimuli. As a stimulus set for practices, four stimuli with different tumor position, i.e., two far positions $(+5$ and $+15 \mathrm{~mm})$ from the lateral center of the stimuli and two near positions $(-5$ and $-15 \mathrm{~mm}$ ), were prepared. An additional set of five stimuli $(-20,-10,0,+10$, and $+20 \mathrm{~mm})$ was prepared for the localization trials. Fig. 5(c) shows the camera image of the center of the five stimuli.

\subsection{Setup}

Fig. 6 shows the setup for the localization experiment. It consisted of a laparoscopic training box (Endowork-Pro II, Kyoto Kagaku Co., Ltd.), a camera, a monitor for displaying the camera image, a $12-\mathrm{mm}$ trocar (ENDOPATH XCEL

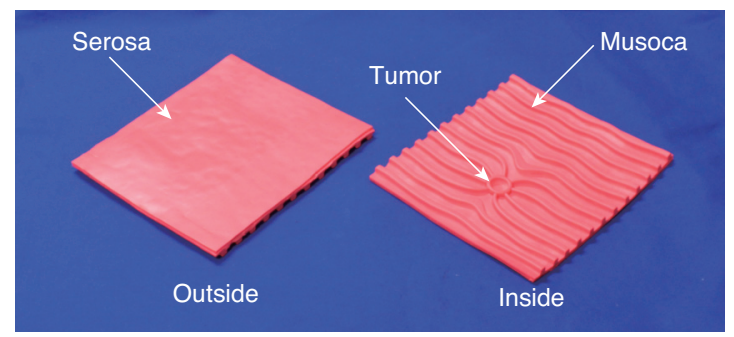

(a)

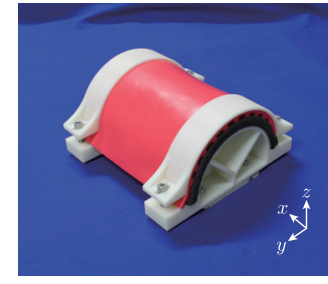

(b)

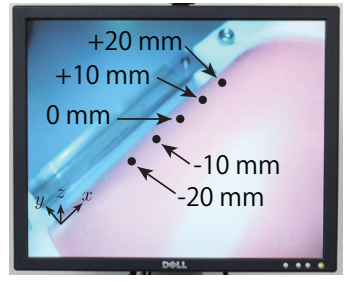

(c)
Fig. 5. (a) Phantom of stomach wall with tumor. (b) Stimulus for localization test. (c) Camera image of stimulus and five tumor positions presented in localization trials. Black dots show the center of the tumor at each position. 
CB12LT, Ethicon Inc.), the tactile sensor, and the tactile ring. The participants were asked to wear headphones playing white noise to avoid hearing sound from the pneumatic drive unit. They manipulated the tactile sensor with their dominant hand and wore the tactile ring on their middle finger of the dominant hand. For the left-handed participant, the setup was symmetrically rearranged. To avoid visual localization of the tumor, the training box was covered with a black cloth and the camera image was blurred slightly. A video detailing the setup and how a participant scanned the stimulus can be found in the supplementary material.

The stimuli were placed on a three-axis force sensor (Gamma SI-32-2.5, ATI Industrial Automation, Inc.), and they were fixed to the inside of the training box. Exerted force was measured at sampling frequencies of $1 \mathrm{kHz}$. If the participants applied a larger force than $1 \mathrm{~N}$, an auditory alarm (1 kHz, square wave) was transmitted to the participant through the headphones. The participants were asked to reduce the force if they heard the alarm. The force limitation was to prevent an overload on the stimulus and also imitated real-life conditions since surgeons do not apply a large amount of force to the tissue in clinical situations. As a tool to indicate the position of the tumor, a toroidal marker that has the same diameter as the tumor was prepared. The marker was magnetically connected to a linear encoder that measures its lateral position; thus, the estimated tumor position by the participants was measured through the position of the marker. While the participants scanned the stimulus surface, the marker was removed from the training box.

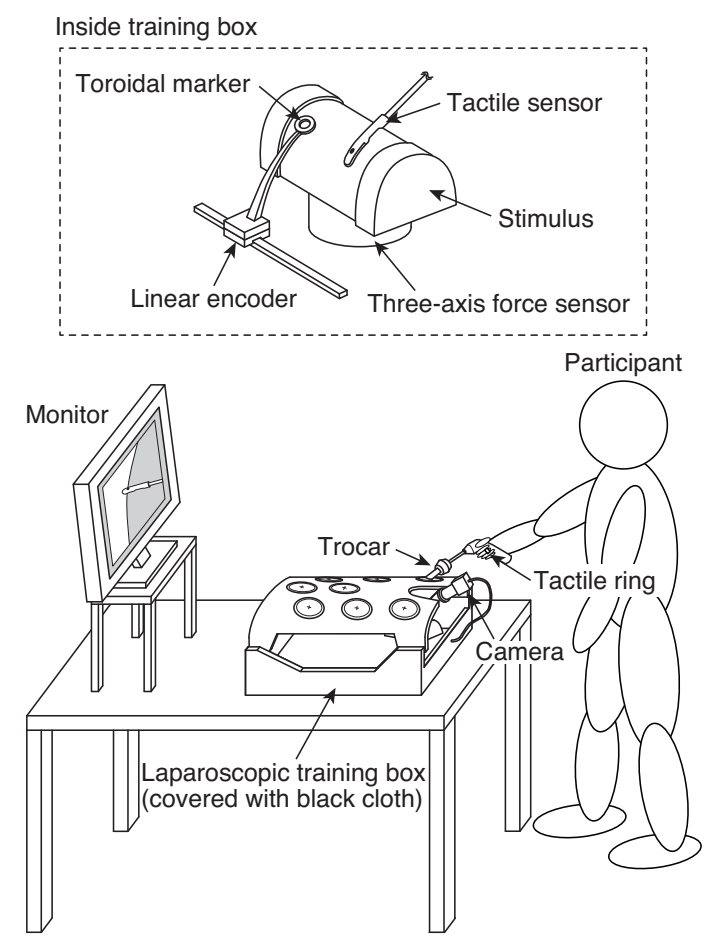

Fig. 6. Setup for the localization test. The dashed rectangle details the inside of the training box. For the left-handed participant, the stimulus was symmetrically rearranged.

\subsection{Experimental procedure}

The entire experimental procedure consisted of three days for each participant. Fig. 7 shows an example of the detailed procedure. The first day was used for the JND measurements and for practice to become familiar with the sensor manipulation and localization tasks. The remaining two days were used for localization tasks with two experimental conditions. The conditions were set regarding the feedback from the sensor; no tactile feedback condition $(\mathrm{N})$ and tactile feedback condition (T). Under condition N, the participants did not receive any feedback from the sensor. Under condition $\mathrm{T}$, the participants received the pressure stimulation from the tactile ring according to the sensor output. The time interval of each condition was more than 24 hours to reduce learning effects and the participants completed the entire experiment within three weeks. The participants wore the tactile ring regardless of the condition. The experiment took $144 \pm 16 \mathrm{~min}, 101 \pm 16 \mathrm{~min}$, and $98 \pm 22 \mathrm{~min}$ for Days 1,2 , and 3 , respectively.

\subsubsection{JND measurements}

The participants wore the tactile ring but did not manipulate the tactile sensor. Three pressures $(22.7,42.1$, and $61.4 \mathrm{kPa})$ were chosen as reference pressures on the basis of the pressure range of the pneumatic drive unit. The reference pressure was presented to the participant for a couple of seconds; then, the pressure was gradually increased/decreased from the reference point and at the same time, an experimenter gave a cue to the participants. The participants were asked to indicate when they felt a pressure change, and the pressure difference from the reference was recorded as a JND. Each ascending/descending series was alternately conducted six times for each reference pressure. The pre-

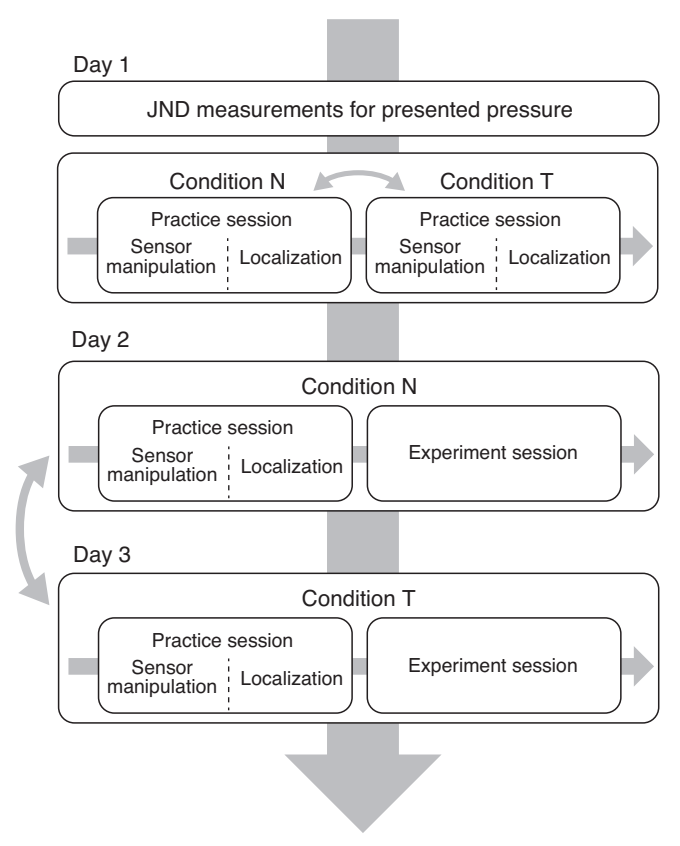

Fig. 7. Example of the experimental procedure for each participant. The time interval between each experimental day was more than 24 hours, and the entire procedure was completed within three weeks. Curved arrows indicate that the order of conditions was counterbalanced across the participants. 
senting order of the three references was counterbalanced across the participants.

\subsubsection{Localization experiment}

After the JND measurements on the first experimental day, the participants practiced the sensor manipulation and localization trials for each condition. The participants were informed that the dimensions of the tumor were always the same and that only the lateral position of the tumor was changed. They were asked to manipulate the sensor as follows. They first made contact with the stimulus, and then laterally slid the sensor in a single direction. After sliding the sensor, they lifted up the sensor. They were instructed not to slide the sensor back and forth. These instructions regarding the manipulation were provided to control the participants' scanning strategy during the localization trials to ensure a fair comparison across the participants.

The participants were forced to practice for at least $4 \mathrm{~min}$ against the stimulus with the tumor located at the lateral center $(0 \mathrm{~mm})$, and they were given the correct position of the tumor with the toroidal marker before the practice. If they wanted more practice time, they were allowed to practice for an additional $1 \mathrm{~min}$. Then, each tumor position from $-15,-5,+5$, and $+15 \mathrm{~mm}$ was randomly presented, and the participants were forced to practice in the same way.

After the participants practiced the sensor manipulation, they had eight practice localization trials. Two of each tumor position from $-15,-5,+5$, and $+15 \mathrm{~mm}$ were randomly presented. The participants were asked to localize the position of the tumor as quickly and accurately as possible within $180 \mathrm{~s}$ in each trial. When they found the position of the tumor or reached the time limit, they were asked to indicate the estimated tumor position with the tip of the sensor and grade confidence in their estimated position on a scale between 1 (not confident at all) to 100 (very confident). The response time was measured with a stopwatch. The toroidal marker was put on the position indicated by the participants with the sensor tip. Then, the marker was shifted to the true position to give the participants feedback about their setting.

Each subsequent experimental day consisted of a practice session and an experimental session. The practice session was similar to practices in the first day; however, the participants were forced to practice sensor manipulation for at least $2 \mathrm{~min}$ for five tumor positions (the tumor position of $0 \mathrm{~mm}$ followed by positions of $-15,-5,+5$, and $+15 \mathrm{~mm}$ ). Then, four practices for tumor localization with feedback about the correctness of their setting were conducted (one for each randomly presented position from $-15,-5,+5$, and $+15 \mathrm{~mm}$ ). In the experimental session, the participants conducted the localization trial 20 times, for four of the randomly presented tumor positions from $-20,-10,0,+10$, and $+20 \mathrm{~mm}$. The protocol was the same as that for the localization practice; however, the participants did not receive any feedback about the true tumor position.

\subsection{Data analysis}

\subsubsection{Localization performances}

The absolute error between the estimated and the true position of the tumor, the confidence rating, and the response time were recorded for each localization trial. As an average value of localization performance for each participant, the median of each parameter was calculated instead of the mean because it was found that the raw data were not normally distributed according to Shapiro-Wilk tests.

To quantitatively assess how well the participants localized the tumor, we calculated an expected value of the absolute error for a random tumor localization. Let $L$ be the maximum lateral length that can be scanned by the tactile sensor, and $x_{\mathrm{t} j},(j=1,2, \cdots, 5)$ be the prepared positions of the tumor. At first, the length $L$ was divided into a large number of $N$ segments. We assumed that the participants randomly chose one segment from $N$ discrete segments, and the probability of segment selection was uniform within $L$. The probability that one of each tumor position is randomly presented, and one of each discrete segment is randomly selected is $1 /(5 N)$. In this case, the expected value $E$ is calculated according to the definition that a summation of each product between a probability and an outcome is as follows:

$$
\begin{aligned}
E & =\lim _{N \rightarrow \infty} \sum_{i=0}^{N} \sum_{j=1}^{5} \frac{\Delta x}{5 L}\left|x_{i}-x_{\mathrm{t} j}\right| \\
& =\frac{1}{5 L} \sum_{j=1}^{5} \int_{0}^{L}\left|x_{i}-x_{\mathrm{t} j}\right| \mathrm{d} x \\
& =\frac{1}{5 L} \sum_{j=1}^{5} x_{\mathrm{t} j}^{2}-\frac{1}{5} \sum_{j=1}^{5} x_{\mathrm{t} j}+\frac{L}{2},
\end{aligned}
$$

where $x_{i}=(L / N) i$ is the position of the $i$-th discrete segment $(i=0,1, \cdots, N)$ from the origin, and $\Delta x=L / N$ is the width of each discrete segment, as shown in Fig. 8. The length that can be indicated $(L)$ is $80 \mathrm{~mm}$ because the entire length is $100 \mathrm{~mm}$, and the diameter of the tumor is $20 \mathrm{~mm}$. The tumor positions are 20,30,40,50, and $60 \mathrm{~mm}$ from the left side (corresponding to $-20,-10,0,+10$, and $+20 \mathrm{~mm}$ from the center). We substituted these values into Eq. (4) and obtained an expected value of absolute error as $22.5 \mathrm{~mm}$.

\subsubsection{Statistical test}

Effects of the tactile feedback and the tumor position on the performance indices were investigated through statistical tests. The significance level was set to 0.05 . Before analyses, a Shapiro-Wilk test was conducted to check whether all the dependent parameters were distributed normally. For each index in all participants, a two-way repeated measures ANOVA with the conditions ( $\mathrm{N}$ and $\mathrm{T}$ ) and the tumor positions $(-20,-10,0,+10$, and $+20 \mathrm{~mm})$ as factors was conducted. Before the ANOVAs, a Mauchly's sphericity test

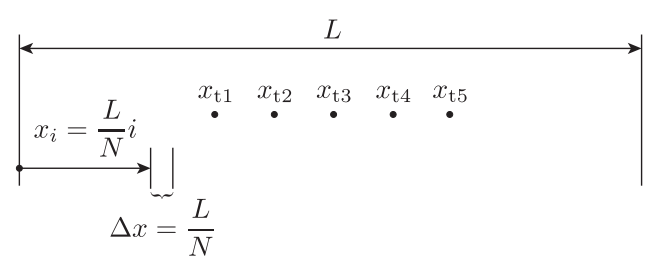

Fig. 8. Calculation of expected value of absolute localization error. 
was conducted to confirm the assumption of the homogeneity of the variance. If the assumption of the homogeneity of the variance was violated, the Greenhouse-Geisser correction was applied. In the case where a significant influence of the tumor position was shown, post-hoc paired $t$-tests for all possible combinations of the tumor positions were conducted with Bonferroni correction. If the assumption of a normal distribution was violated, we used the aligned rank transform [46] for non-parametric factorial analyses before the ANOVAs. If a significant influence of the tumor position was confirmed, post-hoc Wilcoxon signed rank tests for all possible combinations were conducted with Bonferroni correction.

\section{Results}

\subsection{Weber ratio}

Fig. 9(a) shows the result of JND measurements. The open circles and error bars show the mean and standard deviation of the JNDs for 12 participants at each reference, respectively. A linear function was fitted to the mean JNDs to reveal the Weber ratio of the pressure (the dashed line in Fig. 9(a)). The slope of the fitted line is 0.063 , and the intercept is 7.8. The coefficient of determination is 0.999 . We also directly calculated the Weber ratio (each JND was divided by the corresponding reference) for each reference (see Fig. 9(b)). The dashed curve was drawn on the basis of

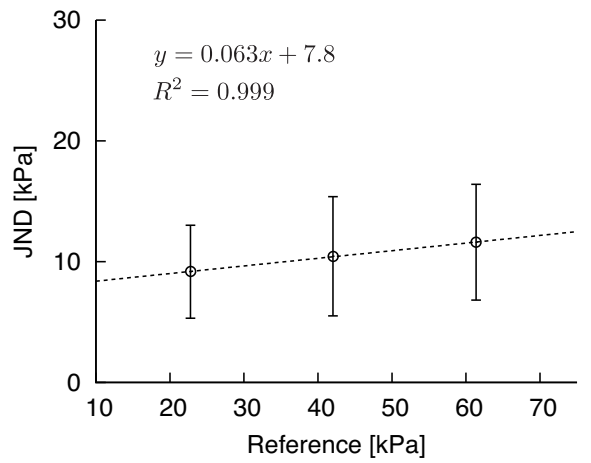

(a)

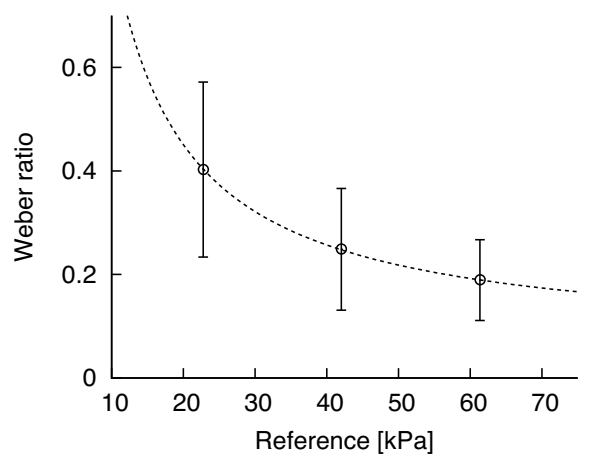

(b)

Fig. 9. Results of JND measurements. Open circles and error bars indicate the means and standard deviations of the data for 12 participants, respectively. (a) JND for reference pressure. The dashed line shows the fitted linear function. (b) Weber ratio calculated from the JNDs for each reference. The dashed curve was drawn from coefficients of the linear function. coefficients for the fitted linear function in Fig. 9(a). It was observed that the Weber ratio decreased according to the increasing reference pressure. The Weber ratio was at least 0.40 at the reference pressure of $22.7 \mathrm{kPa}$.

\subsection{Localization performances}

Fig. 10 shows the absolute error, the confidence in the participants' answer, and the response time for the presented tumor positions under each experimental condition. Fig. 11 shows the performance indices for each participant under each condition.

An ANOVA showed a significant influence of the condition on the absolute error $(F(1,11)=6.2, p=0.030)$. This result indicates that the mean absolute error for condition $\mathrm{T}$ is significantly smaller than that for condition $\mathrm{N}$. The ANOVA also showed a significant influence of tumor

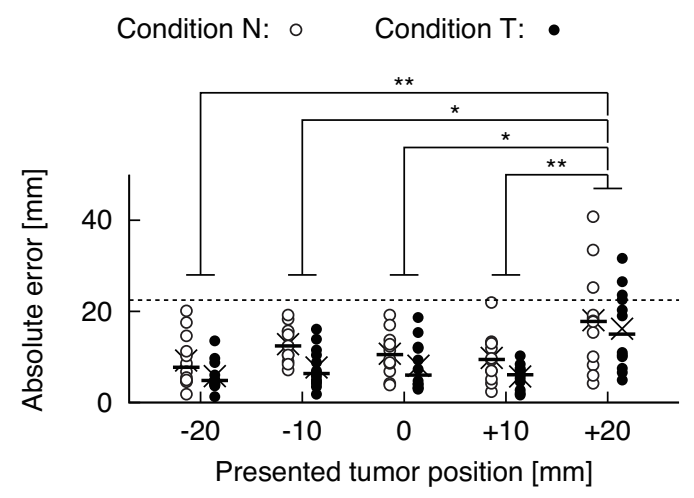

(a)

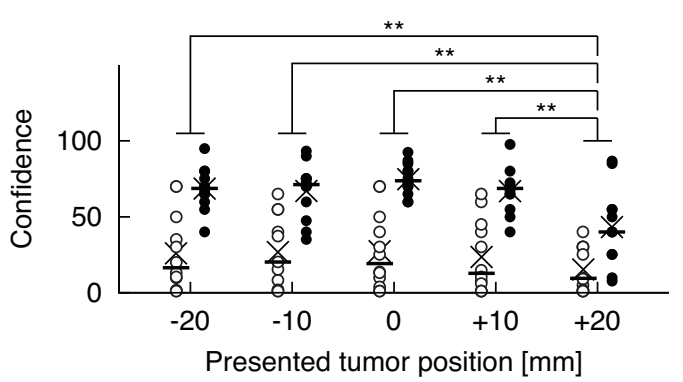

(b)

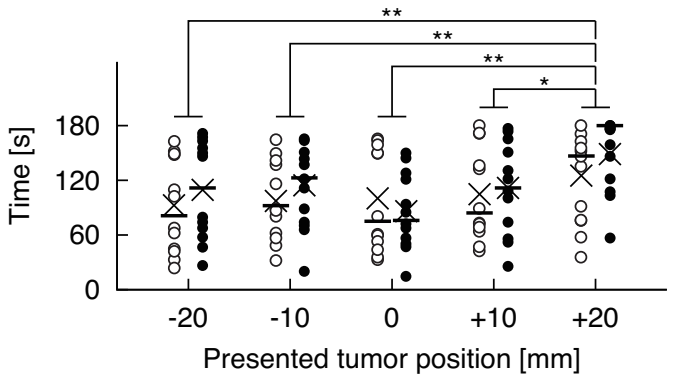

(c)

Fig. 10. Localization performances for the condition and presented tumor position. Open and filled circles indicate the data for the no feedback (N) and the tactile feedback (T) conditions, respectively. The results for 12 participants are shown. Cross marks and horizontal lines show the means and medians of the data, respectively. * indicates $p<0.05$ and ${ }^{* *} p<0.01$ with post-hoc $t$-tests or Wilcoxon signed rank tests with Bonferroni correction. (a) Absolute error. The dashed line shows the expected value of random localization. (b) Confidence of participant's answer. (c) Response time. 


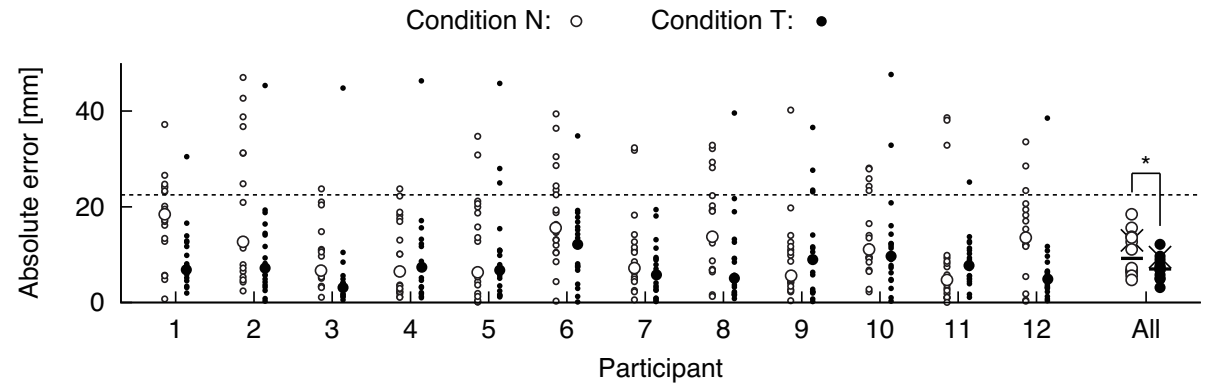

(a)

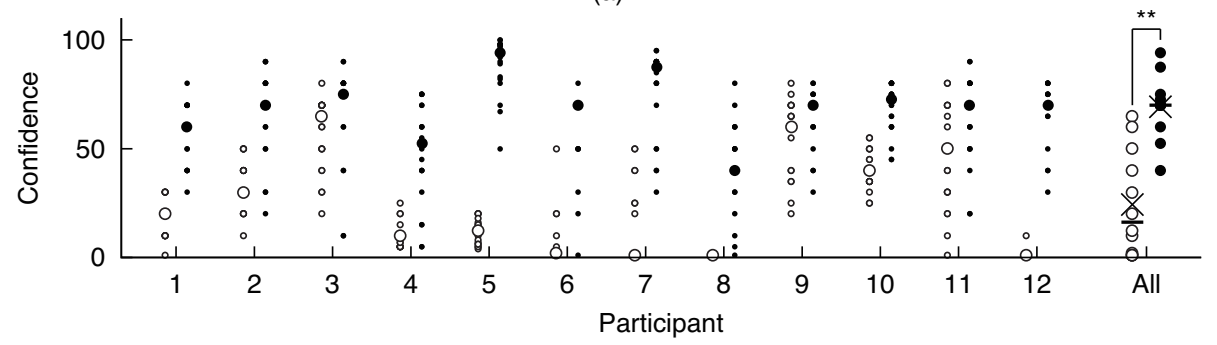

(b)

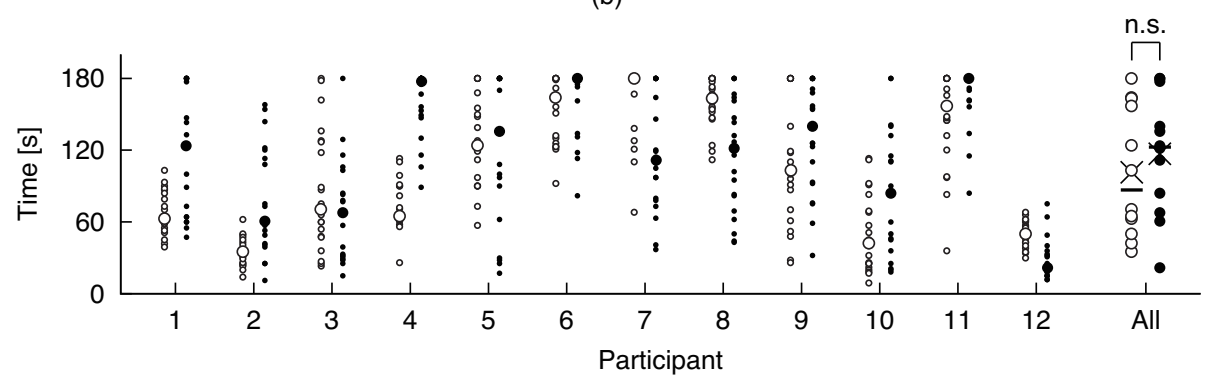

(c)

Fig. 11. Localization performances of individual participants. Open and filled circles indicate the data for the no feedback $(\mathrm{N})$ and the tactile feedback (T) conditions, respectively. Small circles show the raw data for 20 localization trials. Large circles show the median of the raw data. On the right, the median of the data for each participant is summarized. Cross marks and horizontal lines show means and medians of the summarized data, respectively. * indicates $p<0.05$ and ${ }^{* *} p<0.01$ with ANOVAs on condition. (a) Absolute error. The dashed line shows the expected value for random localization. (b) Confidence of participant's answer. (c) Response time.

position on the error $\left(F(2.5,28)=14, p=2.3 \times 10^{-5}\right)$. Paired $t$-tests showed significant differences between tumor positions -20 and $+20 \mathrm{~mm}\left(p=7.2 \times 10^{-4}\right),-10$ and $+20 \mathrm{~mm}(p=0.049), 0$ and $+20 \mathrm{~mm}(p=0.015)$, and +10 and $+20 \mathrm{~mm}\left(p=3.2 \times 10^{-4}\right)$. These results indicate that the mean absolute error for the localization of the tumor at $+20 \mathrm{~mm}$ is significantly larger than for other positions. There were no significant interactions between the condition and tumor position $(F(1.6,17)=0.28, p=0.71)$.

An ANOVA with the aligned rank transform showed a significant influence of the condition on the participants' confidence $\left(F(1,99)=190, p=2.2 \times 10^{-16}\right)$. This result indicates that the median of the confidence for condition $\mathrm{T}$ is significantly greater than that for condition N. The ANOVA also showed a significant influence of tumor position on the confidence $\left(F(4,99)=7.0, p=5.0 \times 10^{-5}\right)$. Wilcoxon signed rank tests showed significant differences between tumor positions -20 and $+20 \mathrm{~mm}(p=0.0057),-10$ and $+20 \mathrm{~mm}\left(p=4.4 \times 10^{-4}\right), 0$ and $+20 \mathrm{~mm}\left(p=4.8 \times 10^{-6}\right)$, and +10 and $+20 \mathrm{~mm}\left(p=6.7 \times 10^{-4}\right)$. These results indicate that the median of confidence for the tumor at $+20 \mathrm{~mm}$ is significantly smaller than for other positions. There were no significant interactions $(F(4,99)=1.8, p=0.13)$.

An ANOVA with the aligned rank transform showed no significant influence of the condition on the response time $(F(1,99)=3.2, p=0.078)$. The ANOVA also showed a significant influence of tumor position on the time $\left(F(4,99)=7.1, p=4.9 \times 10^{-5}\right)$. Wilcoxon signed rank tests showed significant differences between tumor positions -20 and $+20 \mathrm{~mm}(p=0.0021),-10$ and $+20 \mathrm{~mm}$ $\left(p=4.8 \times 10^{-6}\right), 0$ and $+20 \mathrm{~mm}\left(p=2.1 \times 10^{-4}\right)$, and +10 and $+20 \mathrm{~mm}(p=0.010)$. These results indicate that the median of time for $+20 \mathrm{~mm}$ is significantly larger than for other positions. There were no significant interactions $(F(4,99)=1.3, p=0.27)$.

\section{Discussion}

We will firstly discuss the performance of the tactile ring for presentation of pressure. The Weber ratio of pressure was 0.40 at the reference pressure of $22.7 \mathrm{kPa}$. This value is comparable to the value obtained for the similar tactile device that presents a force to the user's finger pad [37]. However, Fig. 9(b) shows that the Weber ratio is not constant for the reference pressures; thus, Weber's law does not hold for the pressure presented by the tactile ring. Similar behavior of the Weber ratio has also been reported in intensity discrimination of sound [47]. This is referred to as a near 
miss to Weber's law. The modification of Weber's law is described as follows [48]:

$$
\Delta I=k\left(I+I_{0}\right)
$$

where $\Delta I$ is the JND of a stimulus, $I$ is the reference stimulus, $k$ is the Weber ratio, and $I_{0}$ is a constant value. This equation indicates that if the reference stimulus is larger than $I_{0}$, the JND is almost proportional to the reference. Alternatively, if the reference has a value close to $I_{0}$, the JND is also affected by $I_{0}$. The existence of $I_{0}$ might be due to noise during the perceptual process [48]. In this study, the diaphragm pump is a possible source of a perceptual noise. The pump supplies pressure with subtle pulsation due to its driving mechanism. When the pressure intensity is relatively small, the subtle pulsative pressure might degrade the perception of the change in pressure. If the pulsative pressure could be reduced, the Weber ratio might not increase even for smaller reference stimuli.

The tactile ring always presented an offset pressure of $15.8 \mathrm{kPa}$ as described in Section 4.2. The offset was added to allow for the presentation of the negative feedback direction. The offset should not be large because a large pressure results in a smaller dynamic response of the pneumatic drive unit as shown in Fig.3(d) and might also result in lowered sensitivity to pressure change due to adaptation [49], [50]. The empirically determined offset might actually lie within an appropriate range considering the factors mentioned above. Moreover, it can be seen that the Weber ratio was 0.55 for $15.8 \mathrm{kPa}$ in Fig. 9(b). The typical feedback obtained by the user while scanning the phantom tumor is shown in Fig. 4(b). The ratio between the peak-to-peak value that marks the tumor (about $16.7 \mathrm{kPa}$ from Fig. 4(b)) and the offset pressure $(15.8 \mathrm{kPa})$ was 1.06 . Thus, the pressure change due to the tumor was sufficiently perceivable, and it indicates that the gain applied to the sensor output was appropriate.

Next, we will discuss how the tactile ring contributes to tumor localization. The localization error was significantly reduced by providing tactile feedback. Although the tactile feedback gave only simple information to the user, it was effective in intraoperative tumor localization in laparoscopic surgery. Fig.11(a) shows that the median of the error for each participant lies below the estimated maximal value of $22.5 \mathrm{~mm}$; thus, the participants performed better than random localizations even under condition $\mathrm{N}$. This implies that the participants could utilize other information such as direct haptic information or visual information from the camera monitor. These results are similar to those obtained in our previous research [33], where participants achieved better detection performance (discrimination between phantoms with/without the tumor) than chance level under the no feedback condition. This might imply that direct haptic information and visual information are somewhat informative in detection, but they cannot largely contribute to the localization. Tactile feedback, however, contributed to the localization. This might be because of the high wearability of the tactile ring and the spatial coincidence between the manipulating hand and the feedback site. The high wearability and the spatial coincidence might have effectively facilitated the sensory-motor integration and the interpretation of the tactile feedback for tumor localization.
Tactile feedback is also effective in the enhancement of the participants' confidence. Although the localization errors for some participants were not reduced by providing tactile feedback, the confidence was enhanced for all participants. The participants could receive direct haptic information and visual information in condition $\mathrm{N}$; however, they could not fully rely on this information to make decisions. Tactile feedback also contributed to the reliability of the participants' decisions. Tactile feedback had no significant effect on response time. This might be because of the different localization strategies used by the various participants. For instance, under condition $\mathrm{N}$, some participants fully used the maximum time because they could not receive any reliable information. On the other hand, some participants stopped the manipulation early when they could not find the tumor.

In this study, we focused on temporal information-based palpation, in which only a single sensor output was measured and instantaneously fed back to the user. The main characteristic of temporal information-based palpation is that the user should dynamically scan the target surface. Fig. 10(a) shows that the localization error for the tumor at $+20 \mathrm{~mm}$ was significantly greater than that for other positions. In addition, the confidence (the response time) for the tumor at $+20 \mathrm{~mm}$ was significantly smaller (longer) than those for the other tumor positions. This indicates that the participants achieved worse localization performance for the tumor located at the farthest position. This might be due to the loss of depth information in the camera image; that is, the resolution of position information on the monitor is more reduced for the farther position as shown in Fig. 5(c). The appropriate manipulation of the sensor might be difficult for the participants at the farther position. On the other hand, the surgeon can move the target tissue so that they could appropriately scan the target surface; thus, the worsened localization error due to scanning the farther position might be reduced in surgical situations. In future work, we will investigate how well laparoscopic surgeons conduct the tumor localization using our temporal information-based palpation system in an in-vivo situation.

Finally, we will discuss advantages and issues in clinical applications. The tactile ring can be used in a disposable manner because of its low fabrication cost. The tactile ring does not include any electrical components, which is an advantage for sterilization. We confirmed that the tactile ring can be sterilized by ethylene oxide gas and its performance is not affected by the sterilization. We have also confirmed that our acoustic tactile sensor can be sterilized. Thus, this study shows a laparoscopic palpation system with high clinical applicability. The tactile ring also has high wearability owing to the design of the rigid ring body, and the surgeon can easily wear it by inserting the ring onto their finger. On the other hand, the tactile ring still has a few issues. For instance, an expert surgeon commented that the pulsative pressure always presented by the tactile ring causes some confusion with the pressure difference due to the tumor. This issue will be addressed in future studies. Another issue that still needs attention is that the signal processing implemented to control the tactile ring was determined empirically. Currently, there is no systematic method to optimize parameters such as the values of the 
gain and offset. In future work, we will investigate how these parameters affect performance in tumor localization.

\section{Conclusion}

In this study, we developed and evaluated a pneumatic tactile ring, which is a clinically applicable tactile device to provide instantaneous sensory feedback in laparoscopic tumor localization. We designed a ring-type device to achieve high wearability. The pneumatic tactile ring has high applicability to surgical situations because it is lightweight, costeffective, sterilizable, and disposable. We also developed a pneumatic drive unit that consists of a diaphragm pump and a voice coil motor to supply the pressure to the tactile ring. The fundamental analysis of the tactile ring revealed that the inner pressure could be controlled linearly based on the input current to the voice coil motor, and it has the bandwidth of at least $1.3 \mathrm{~Hz}$. Moreover, a psychophysical experiment was also conducted to assess the pressure that could be distinguished by the user and how the sensory feedback through the tactile ring contributes to tumor localization performance. It was found that the Weber ratio of the pressure is at least 0.40 . Moreover, the tactile feedback significantly reduced the localization error and improved participants' confidence in their estimation of the tumor position. Thus, the tactile ring is effective for intraoperative tumor localization in laparoscopic surgery.

\section{ACKNOWLEDGMENTS}

This work was supported in part by JSPS KAKENHI Grant numbers JP16J08253 and JP17H01252.

\section{REFERENCES}

[1] K. Hoyt, B. Castaneda, M. Zhang, P. Nigwekar, P. A. di Sant'agnese, J. V. Joseph, J. Strang, D. J. Rubens, and K. J. Parker, "Tissue elasticity properties as biomarkers for prostate cancer," Cancer Biomark, vol. 4, no. 4-5, pp. 213-225, 2008.

[2] Y. Zhao, T. B. Zhang, C. H. Bao, X. X. Chen, Y. Wang, and Q. Wang, "Physical properties of gastrointestinal stromal tumors based on atomic force microscope analysis," Genetics and Molecular Research, vol. 12, no. 4, pp. 5774-5785, 2013.

[3] S. Kawano, M. Kojima, Y. Higuchi, M. Sugimoto, K. Ikeda, N. Sakuyama, S. Takahashi, R. Hayashi, A. Ochiai, and N. Saito, "Assessment of elasticity of colorectal cancer tissue, clinical utility, pathological and phenotypical relevance," Cancer Science, vol. 106, no. 9, pp. 1232-1239, 2015.

[4] M. Li, H. Liu, A. Jiang, L. D. Seneviratne, P. Dasgupta, K. Althoefer, and $\mathrm{H}$. Wurdemann, "Intra-operative tumour localisation in robotassisted minimally invasive surgery: A review," Proceedings of the Institution of Mechanical Engineers Part H Journal of Engineering in Medicine, vol. 228, pp. 509-522, 2014.

[5] J. Back, P. Dasgupta, L. Seneviratne, K. Althoefer, and H. Liu, "Feasibility study- novel optical soft tactile array sensing for minimally invasive surgery," in IEEE/RSJ International Conference on Intelligent Robots and Systems, 2015, pp. 1528-1533.

[6] M. Beccani, C. D. Natali, L. J. Sliker, J. A. Schoen, M. E. Rentschler, and P. Valdastri, "Wireless tissue palpation for intraoperative detection of lumps in the soft tissue," IEEE Transactions on Biomedical Engineering, vol. 61, no. 2, pp. 353-361, 2014.

[7] P. Culmer, J. Barrie, R. Hewson, M. Levesley, M. Mon-Williams, D. Jayne, and A. Neville, "Reviewing the technological challenges associated with the development of a laparoscopic palpation device," The International Journal of Medical Robotics and Computer Assisted Surgery, vol. 8, no. 2, pp. 146-159, 2012.

[8] P. Richard and P. Coiffet, "Human perceptual issues in virtual environments: sensory substitution and information redundancy," in Proc. IEEE International Workshop on Robot and Human Communication, 1995, pp. 301-306.
[9] M. Tavakoli, A. Aziminejad, R. V. Patel, and M. Moallem, "Methods and mechanisms for contact feedback in a robot-assisted minimally invasive environment," Surgical Endoscopy And Other Interventional Techniques, vol. 20, no. 10, pp. 1570-1579, 2006.

[10] A. Talasaz and R. V. Patel, "Integration of force reflection with tactile sensing for minimally invasive robotics-assisted tumor localization," IEEE Transactions on Haptics, vol. 6, no. 2, pp. 217-228, 2013.

[11] G. Tholey, J. P. Desai, and A. E. Castellanos, "Force feedback plays a significant role in minimally invasive surgery: Results and analysis," Annals of Surgery, vol. 241, no. 1, pp. 102-109, 2005.

[12] J. C. Gwilliam, M. Mahvash, B. Vagvolgyi, A. Vacharat, D. D. Yuh, and A. M. Okamura, "Effects of haptic and graphical force feedback on teleoperated palpation," in 2009 IEEE International Conference on Robotics and Automation, 2009, pp. 677-682.

[13] C. Pacchierotti, L. Meli, F. Chinello, M. Malvezzi, and D. Prattichizzo, "Cutaneous haptic feedback to ensure the stability of robotic teleoperation systems," The International Journal of Robotics Research, vol. 34, no. 14, pp. 1773-1787, 2015.

[14] C. H. King, M. O. Culjat, M. L. Franco, C. E. Lewis, E. P. Dutson W. S. Grundfest, and J. W. Bisley, "Tactile feedback induces reduced grasping force in robot-assisted surgery," IEEE Transactions on Haptics, vol. 2, no. 2, pp. 103-110, 2009.

[15] M. Li, S. Luo, T. Nanayakkara, L. D. Seneviratne, P. Dasgupta, and K. Althoefer, "Multi-fingered haptic palpation using pneumatic feedback actuators," Sensors and Actuators A: Physical, vol. 218, pp. $132-141,2014$.

[16] S. Yun, J. Yoo, S. Lim, J. Park, H.-K. Lee, and K.-S. Yun, “Threeaxis pneumatic tactile display with integrated capacitive sensors for feedback control," Microsystem Technologies, vol. 22, no. 2, pp. 275-282, 2016.

[17] M. Li, T. Ranzani, S. Sareh, L. D. Seneviratne, P. Dasgupta, H. A. Wurdemann, and K. Althoefer, "Multi-fingered haptic palpation utilizing granular jamming stiffness feedback actuators," Smart Materials and Structures, vol. 23, no. 9, p. 095007, 2014.

[18] M. Bianchi, J. C. Gwilliam, A. Degirmenci, and A. M. Okamura, "Characterization of an air jet haptic lump display," in Proc. Annual International Conference of the IEEE Engineering in Medicine and Biology Society, 2011, pp. 3467-3470.

[19] C. R. Wagner, S. J. Lederman, and R. D. Howe, "A tactile shape display using rc servomotors," in Proc. Symposium on Haptic Interfaces for Virtual Environment and Teleoperator Systems, 2002, pp. 354-355.

[20] R. L. Feller, C. K. L. Lau, C. R. Wagner, D. P. Perrin, and R. D. Howe, "The effect of force feedback on remote palpation," in Proc. IEEE International Conference on Robotics and Automation, vol. 1, 2004, pp. 782-788.

[21] J. Hergenhan, H. Alagi, H. Worn, M. Uhl, R. Schirren, and S. Reiser "Prototype of a haptic display for the evaluation of sensible haptic feedback in remote palpation," in Proc. IEEE International Symposium on Medical Measurements and Applications, 2014, pp. 1-6.

[22] C. Roke, A. Spiers, T. Pipe, and C. Melhuish, "The effects of laterotactile information on lump localization through a teletaction system," in Proc. IEEE World Haptics Conference, 2013, pp. 365-370.

[23] C. Pacchierotti, D. Prattichizzo, and K. J. Kuchenbecker, "Cutaneous feedback of fingertip deformation and vibration for palpation in robotic surgery," IEEE Transactions on Biomedical Engineering, vol. 63, no. 2, pp. 278-287, 2016.

[24] R. D. Howe, W. J. Peine, D. A. Kantarinis, and J. S. Son, "Remote palpation technology," IEEE Engineering in Medicine and Biology Magazine, vol. 14, no. 3, pp. 318-323, 1995.

[25] N. A. Mansour, A. M. R. F. El-Bab, and M. Abdellatif, "Design of a novel multi-modal tactile display device for biomedical applications," in Proc. IEEE RAS EMBS International Conference on Biomedical Robotics and Biomechatronics, 2012, pp. 183-188.

[26] E. P. Scilingo, N. Sgambelluri, D. D. Rossi, and A. Bicchi, "Haptic displays based on magnetorheological fluids: design, realization and psychophysical validation," in Proc. Symposium on Haptic Interfaces for Virtual Environment and Teleoperator Systems, 2003, pp. $10-15$.

[27] J. S. Oh, J. K. Kim, S. R. Lee, S. B. Choi, and B. K. Song, “Design of tactile device for medical application using magnetorheological fluid," Journal of Physics: Conference Series, vol. 412, no. 1, p. 012047, 2013.

[28] H. Ishizuka and N. Miki, "Development of a tactile display with $5 \mathrm{~mm}$ resolution using an array of magnetorheological fluid," Japanese Journal of Applied Physics, vol. 56, no. 6S1, p. 06GN19, 2017. 
[29] P. Goethals, H. Lintermans, M. M. Sette, D. Reynaerts, and H. Van Brussel, "Powerful compact tactile display with microhydraulic actuators," in Proc. EuroHaptics 2008: Haptics: Perception, Devices and Scenarios, M. Ferre, Ed. Berlin, Heidelberg: Springer Berlin Heidelberg, 2008, pp. 447-457.

[30] H.-Y. Yao, V. Hayward, and R. E. Ellis, "A tactile enhancement instrument for minimally invasive surgery," Computer Aided Surgery, vol. 10, no. 4, pp. 233-239, 2005.

[31] D. D. Lorenzo, Y. Koseki, E. D. Momi, K. Chinzei, and A. M. Okamura, "Experimental evaluation of a coaxial needle insertion assistant with enhanced force feedback," in Proc. Annual International Conference of the IEEE Engineering in Medicine and Biology Society, 2011, pp. 3447-3450.

[32] Y. Tanaka, T. Fukuda, M. Fujiwara, and A. Sano, "Tactile sensor using acoustic reflection for lump detection in laparoscopic surgery," International Journal of Computer Assisted Radiology and Surgery, vol. 10, no. 2, pp. 183-193, 2015.

[33] T. Fukuda, Y. Tanaka, A. M. L. Kappers, M. Fujiwara, and A. Sano, "Visual and tactile feedback for a direct-manipulating tactile sensor in laparoscopic palpation," The International Journal of Medical Robotics and Computer Assisted Surgery, vol. 14, no. 2, 2018, e1879.

[34] C. Pacchierotti, S. Sinclair, M. Solazzi, A. Frisoli, V. Hayward, and D. Prattichizzo, "Wearable haptic systems for the fingertip and the hand: Taxonomy, review, and perspectives," IEEE Transactions on Haptics, vol. 10, no. 4, pp. 580-600, 2017.

[35] K. Minamizawa, S. Fukamachi, H. Kajimoto, N. Kawakami, and S. Tachi, "Gravity grabber: Wearable haptic display to present virtual mass sensation," in Proc. ACM SIGGRAPH 2007 Emerging Technologies. New York, NY, USA: ACM, 2007.

[36] M. Bianchi and A. Serio, "Design and characterization of a fabricbased softness display," IEEE Transactions on Haptics, vol. 8, no. 2, pp. 152-163, 2015.

[37] G. Frediani, D. Mazzei, D. E. De Rossi, and F. Carpi, "Wearable wireless tactile display for virtual interactions with soft bodies," Frontiers in Bioengineering and Biotechnology, vol. 2, p. 31, 2014.

[38] T. Aoki, H. Mitake, D. Keoki, S. Hasegawa, and M. Sato, “Wearable haptic device to present contact sensation based on cutaneous sensation using thin wire," in Proceedings of the International Conference on Advances in Computer Enterntainment Technology, ser. ACE '09. New York, NY, USA: ACM, 2009, pp. 115-122.

[39] G. A. Pradana, A. D. Cheok, M. Inami, J. Tewell, and Y. Choi, "Emotional priming of mobile text messages with ring-shaped wearable device using color lighting and tactile expressions," in Proceedings of the 5th Augmented Human International Conference, ser. AH '14. New York, NY, USA: ACM, 2014, pp. 14:1-14:8.

[40] S. Je, O. Choi, K. Choi, M. Lee, H.-J. Suk, L. Chan, and A. Bianchi, "Designing skin-dragging haptic motions for wearables," in Proceedings of the 2017 ACM International Symposium on Wearable Computers, ser. ISWC '17. New York, NY, USA: ACM, 2017, pp. 98-101.

[41] C. Pacchierotti, G. Salvietti, I. Hussain, L. Meli, and D. Prattichizzo, "The hring: A wearable haptic device to avoid occlusions in hand tracking," in 2016 IEEE Haptics Symposium (HAPTICS), 2016, pp. 134-139.

[42] A. Russomanno, R. B. Gillespie, S. O'Modhrain, and J. Barber, "Modeling pneumatic actuators for a refreshable tactile display," in Proc. EuroHaptics 2014: Haptics: Neuroscience, Devices, Modeling, and Applications, M. Auvray and C. Duriez, Eds. Berlin, Heidelberg: Springer Berlin Heidelberg, 2014, pp. 385-393.

[43] W. J. Peine, P. S. Wellman, and R. D. Howe, "Temporal bandwidth requirements for tactile shape displays," in Proc. IMECE Haptics Symposium, 1997.

[44] S. Coren, The left-hander syndrome: The causes and consequences of left-handedness. New York: McGraw-Hill, 2000.

[45] Japanese Gastric Cancer Association, "Japanese classification of gastric carcinoma: 3rd English edition," Gastric Cancer, vol. 14, no. 2, pp. 101-112, 2011.

[46] J. O. Wobbrock, L. Findlater, D. Gergle, and J. J. Higgins, "The aligned rank transform for nonparametric factorial analyses using only anova procedures," in Proc. the SIGCHI Conference on Human Factors in Computing Systems, 2011, pp. 143-146.

[47] W. Jesteadt, C. C. Wier, and D. M. Green, "Intensity discrimination as a function of frequency and sensation level," The Journal of the Acoustical Society of America, vol. 61, no. 1, pp. 169-177, 1977.

[48] G. A. Gescheider, Psychophysics: The fundamentals. Mahwah, NJ: Lawrence Erlbaum Associates, 1997.
[49] S. J. Bensmaia, Y. Y. Leung, S. S. Hsiao, and K. O. Johnson, "Vibratory adaptation of cutaneous mechanoreceptive afferents," Journal of Neurophysiology, vol. 94, no. 5, pp. 3023-3036, 2005.

[50] Y. G. Chung, S. W. Han, H.-S. Kim, S.-C. Chung, J.-Y. Park, C. Wallraven, and S.-P. Kim, "Adaptation of cortical activity to sustained pressure stimulation on the fingertip," BMC Neuroscience, vol. 16, no. 1, p. 71, 2015.

Tomohiro Fukuda Biography text here.

Yoshihiro Tanaka Biography text here.

Astrid M.L. Kappers Biography text here.

Michitaka Fujiwara Biography text here.

Akihito Sano Biography text here. 